\title{
The Distribution of Partially Exchangeable Random Variables
}

\author{
Hanxiang Peng*,1,2 \\ Indiana University-Purdue University at Indianapolis \\ Xin Dang ${ }^{3,2}$ \\ University of Mississippi \\ Xueqin Wang ${ }^{4}$ \\ Sun Yat-Sen University \\ * Corresponding author: Hanxiang Peng (hpeng@math.iupui.edu)
}

April 30, 2009

\footnotetext{
${ }^{1}$ Department of Mathematical Sciences, Indiana University-Purdue University at Indianapolis, Indianapolis, IN, 46202-3216, USA. Telephone: (317)274-8070. Fax: (317)2743460. Email: hpeng@math.iupui.edu. URL: http://www.math.iupui.edu/ hpeng.

${ }^{2}$ This research is supported by the US National Science Foundation under Grant No. DMS-0707074.

${ }^{3}$ Department of Mathematics, University of Mississippi, University, MS 38677, USA. Telephone: (662)915-7409. Fax: (662)915-2361. Email: xdang@olemiss.edu. URL: http://www. olemiss.edu/ xdang.

${ }^{4}$ School of Mathematics \& Computational Science, Zhongshan School of Medicine, Sun Yat-Sen University, PRC. Email: wxueq@mail.sysu.edu.cn.
} 


\begin{abstract}
In this article, we derive the distribution of partially exchangeable binary random variables, generalizing the distribution of exchangeable binary random variables and hence the binomial distribution. The distribution can also be viewed as a mixture of Markov chains. We introduce rectangular complete monotonicity and show that partial exchangebility can be characterized by rectangular complete monotonicity. The distribution aided with rectangular complete monotonicity can be used to analyze serially correlated data common in many areas of science.
\end{abstract}

Key words and phrases: Complete monotonicity, exchangeability, mixture of Markov chains, partial exchangeability, serial correlation. 


\section{Introduction}

Binary responses commonly arise in many fields of science including tumor recurrences in medical science, baseball hits in sports, occupational status in labor market surveys, and presence of a pathogen in dairy science. It is often that each individual binary sequence exhibits serial dependence. When formulating models of such data, it is natural to allow serial dependence. The literature describing the analysis of correlated binary data is extensive. Different approaches measure different effects of covariates and account for dependence structure in different ways. In this article, we will exploit the perception of partial exchangeability to investigate multiple serially dependent responses.

Introduced by de Finetti in 1938, partial exchangeability was studied by many authors including Diaconis (1988), Diaconis and Freedman (1980a, b, c), Freedman (1962a, b), and Zaman (1984). A sequence of binary random variables is partially exchangeable if the joint distribution of any sub-sequence of the first finite terms is invariant under permutations that keep the initial state and the numbers of transitions from $i$ to $j$ for $i=0,1$ unchanged. A more stringent assumption is exchangeability which requires invariance under any permutation. Also introduced by de Finetti in the 1930's and intensely studied over the past century, exchangeability is meant to capture the notion of symmetry in a collection of random variables and often used as an alternative to independence. Partial exchangeability captures symmetry more generally and grasps dependence in a collection of random variables and can be utilized as a candidate for dependence.

A model in which an individual binary sequence is assumed to be recurrent and partially exchangeable is, according to Diaconis and Freedman (1980a, c), a mixture of Markov chains. Whereas the latter, as pointed out by Quintana and Newton (1998), can be viewed as a random-effects model in which the unobservable latent transi- 
tion matrices follow some unknown distribution. Markov chains and their mixtures are widely used to model serially correlated data for their structural simplicity and interpretational ease. Our proposed distribution can be viewed either as a Markovchain-mixture model (both parametric and nonparametric), a random-effects model, or a Bayesian model. From our viewpoint, the difference among a partial exchangeable model, a mixture distribution, a Bayesian model, and a random-effects model is more for presentation and interpretation and less for mathematical substance.

We represnt the distribution of partially exchangeable binary random variables in moments of the mixing probability measure, an analog to the formula of the distribution of exchangeable binomial random variables by Kendall (1967), Chow and Teicher (1997), George and Bowman (1995), and Dang, et al. (2009) among others. This representation of the distribution demonstrates that a mixture of Markov chains (with either a parametric or nonparametric mixing distribution) is equivalent to a parametric distribution whose number of parameters depends upon the length of the chain of binary random variables. We show that partial exchangebility can be characterized by rectangular complete monotonicity. The distribution aided with rectangular complete monotonicity can be used to analyze serially correlated data which are common in many areas of science. Peng, Garner and Dang (2009) use rectangular complete monotonic functions to obtain a class of parsimonious Markov chain mixtures, and apply the parsimonious mixtures to analyze two real datasets from dairy and medical sciences and have obtained superior results. Their method is related to the procedure used in Dang, Keeton and Peng (2009) in which a unified approach is presented for analyzing exchangeable binary data with an application in developmental toxicity studies. By exploiting partial exchangeability, Quintana and Newton (1998) investigated the assessment of the order of serial dependence for multiple binary responses. Relating to the Pólya sequence from a one-urn model, Quintana and Newton (1999) 
constructed a two-urn model for the mixtures of Markov chains, providing interesting and useful interpretation.

The rest of the article is organized as follows. In Section 2, we derive the distribution of partial exchangeable binary random variables, discuss the sufficient statistics of the distribution, and introduces rectangular complete monotonicity to characterize partial exchangeability. Technical details can be found in the Appendix.

\section{The Partially Exchangeable Model and Rectan- gular Complete Monotonicity}

In this section, we first give the distribution of partially exchangeable binary r.v.'s, followed by rectangular complete monotonicity and characterization of partial exchangeability.

As Diaconis and Freedman (1980a) elaborated, a sequence of binary r.v.'s $B_{0}, B_{1}, \ldots$ are partially exchangeable if for any two binary sequences $\left\{b_{i}: i=0,1, \ldots, n\right\}$ and $\left\{c_{i}: i=0,1, \ldots, n\right\}$ which have the same initial state and the same transition counts,

$$
\mathbb{P}\left(B_{0}=b_{0}, B_{1}=b_{1}, \ldots, B_{n}=b_{n}\right)=\mathbb{P}\left(B_{0}=c_{0}, B_{1}=c_{1}, \ldots, B_{n}=c_{n}\right) .
$$

Recall that a sequence of binary r.v.'s $B_{0}, B_{1}, \ldots$ is recurrent if

$$
P\left(B_{n}=B_{0} \text { for infinitely many } n\right)=1 \text {. }
$$

Diaconis and Freedman (1980a) show that if $B_{0}, B_{1}, \ldots, B_{n}$ are recurrent and partially exchangeable, then the joint probability of them is a mixture of Markov chains. Specifically, it follows from thier (6) that for the binary $\{0,1\}$ state space, there exists a unique probability measure $Q_{b_{0}}$ on $[0,1]^{2}$ such that

$$
P\left(B_{0}=b_{0}, B_{1}=b_{1}, \ldots, B_{n}=b_{n}\right)=\int_{0}^{1} \int_{0}^{1} u^{t_{00}}(1-u)^{t_{01}} v^{t_{11}}(1-v)^{t_{10}} d Q_{b_{0}}(u, v),
$$

where $t_{i j}$ is the number of transitions from $i$ to $j$ for $i, j=0,1$. From this and the binomial formula, we have the following result. 
Theorem 1 Suppose that $B_{0}, B_{1}, B_{2}, \ldots$ are recurrent and partially exchangeable. Then

$$
\mathbb{P}\left(B_{0}=b_{0}, B_{1}=b_{1}, \ldots, B_{n}=b_{n}\right)=\sum_{i=0}^{t_{01}} \sum_{j=0}^{t_{10}}(-1)^{i+j}\left(\begin{array}{c}
t_{01} \\
i
\end{array}\right)\left(\begin{array}{c}
t_{10} \\
j
\end{array}\right) \lambda_{t_{00}+i, t_{11}+j}^{\left(b_{0}\right)},
$$

where $b_{0}, b_{1}, \ldots, b_{n}$ are $\{0,1\}$-valued, and $\lambda_{j, k}^{\left(b_{0}\right)}$ is the joint probability that the transitions $0 \rightarrow 0$ and $1 \rightarrow 1$ in $B_{0}, B_{1}, \ldots, B_{n}$ simultaneously occur $i$ and $j$ times with initial state $b_{0}$, or symbolically,

$\lambda_{i, j}^{\left(b_{0}\right)}=\mathbb{P}\left(B_{0}=b_{0},(0 \rightarrow 0)^{i},(1 \rightarrow 1)^{j}\right), b_{0}=0,1,0 \leq i \leq t_{00}+t_{01}, 0 \leq j \leq t_{11}+t_{10}$.

We refer (2) the partially exchangeable model and denote it by $\operatorname{PE}(\boldsymbol{\lambda})$, where $\boldsymbol{\lambda}=\left\{\lambda_{i, j}^{\left(b_{0}\right)}: b_{0}=0,1, \quad i=0, \ldots, t_{00}+t_{11}, j=0, \ldots, t_{11}+t_{10}\right\}$. Representation (2) is an analog to the formula of the distribution of exchangeable binomial random variables by Kendall (1967), Chow and Teicher (1997), George and Bowman (1995), and Dang, et al. (2009) among others. It is interesting to observe that a (parametric or nonparametric) mixture of Markov chains is a parametric model with a sequence of parameters $\boldsymbol{\lambda}$ in which the number of parameters increases with the length of the binary sequence.

Remark 1 If $B_{0}, \ldots, B_{n}$ are exchangeable, then

$$
\lambda_{j, k}^{\left(b_{0}\right)}=\sum_{i=0}^{j}(-1)^{i}\left(\begin{array}{l}
j \\
i
\end{array}\right) \pi_{k+i}^{\left(b_{0}\right)}, \quad b_{0} \in\{0,1\}, 0 \leq j \leq t_{00}+t_{01}, 0 \leq k \leq t_{11}+t_{10} .
$$

where $\pi_{i}^{\left(b_{0}\right)}=P\left(B_{0}=b_{0}, B_{1}=1, \ldots, B_{i}=1\right), i \geq 0$. Hence

$$
\mathbb{P}\left(B_{1}=b_{1}, \ldots, B_{n}=b_{n}\right)=\sum_{i=0}^{t_{00}+t_{10}}(-1)^{i}\left(\begin{array}{c}
t_{00}+t_{10} \\
i
\end{array}\right) \pi_{t_{01}+t_{11}+i} .
$$

where $\pi_{j}=P\left(B_{0}=1, B_{1}=1, \ldots, B_{j-1}=1\right), j \geq 1$ with $\pi_{0}=1$. This is the probability under exchangeability, see Dang, et al. (2009). Accordingly, the Binomial distribution is a special case of the partial exchangeable model. Further, under exchangeability,

$$
\pi_{j}^{(0)}=\pi_{j}-\pi_{j+1}, \quad \pi_{j}^{(1)}=\pi_{j+1},
$$


where $\pi_{j}=P\left(B_{0}=1, B_{1}=1, \ldots, B_{j-1}=1\right), j \geq 1$ with $\pi_{0}=1$.

Remark 2 By Diaconis and Freedman (1980a), the binary state space in Theorem 1 can be generalized to a multi-state space.

The marginal probabilities $\boldsymbol{\lambda}=\left\{\lambda_{j, k}^{\left(b_{0}\right)}\right\}$ are moments of the mixing probability measure. Specifically,

$$
\lambda_{i j}^{\left(b_{0}\right)}=\int_{0}^{1} \int_{0}^{1} u^{i} v^{j} d Q_{b_{0}}(u, v), \quad b_{0}=0,1, i, j=0,1, \ldots
$$

The momentum problem is a folklore in functional analysis and one-dimensional Hausdorff momentum problem is related to complete monotonicity $(C M)$. Here we extend it to a two-dimensional sequence, which is related to the two dimensional Hausdorff momentum problem. A finite bivariate sequence $\left\{a_{j, k}: 0 \leq j \leq J, 0 \leq k \leq K\right\}$ is said to be (finite) rectangular completely monotone (RCM) if

$$
(-1)^{r_{1}+r_{2}} \Delta_{1}^{r_{1}} \Delta_{2}^{r_{2}} a_{j, k} \geq 0, \quad 0 \leq j+r_{1} \leq J, 0 \leq k+r_{2} \leq K
$$

where $\Delta_{1}, \Delta_{2}$ are the (univariate) marginal difference operators, i.e., $\Delta_{1} a_{j, k}=a_{j+1, k}-$ $a_{j, k}$ and $\Delta_{2} a_{j, k}=a_{j, k+1}-a_{j, k}$ with $\Delta_{1}^{2}=\Delta_{1}\left(\Delta_{1}\right)$ and $\Delta_{1}^{0}=\Delta_{2}^{0}=I$ being the identity operator. It is not difficult to verify the useful expansion

$$
\Delta_{1}^{r} \Delta_{2}^{s} a_{u, v}=\sum_{j=0}^{r} \sum_{k=0}^{s}\left(\begin{array}{l}
r \\
j
\end{array}\right)\left(\begin{array}{l}
s \\
k
\end{array}\right)(-1)^{r+s-j-k} a_{u+j, v+k} \quad r, s, u, v=0,1, \ldots
$$

for a two-dimensional sequence $\left\{a_{j, k}: j, k=0,1, \ldots\right\}$. The above expansion can be used to verify RCM. Applying (6) to (5) for $r=s=1$ gives $a_{u+1, v+1}-a_{u+1, v}-a_{u, v+1}+$ $a_{u, v} \geq 0$, which motivates us to call rectangular.

It is interesting to observe that the marginal probabilities $\boldsymbol{\lambda}=\left\{\lambda_{j, k}^{\left(b_{0}\right)}\right\}$ are comprised of two RCM sequences $\left\{\lambda_{j, k}^{(0)}\right\}$ and $\left\{\lambda_{j, k}^{(1)}\right\}$ satisfying $\lambda_{0,0}^{(0)}+\lambda_{0,0}^{(1)}=1$. Specifically, for $r_{1}, r_{2} \geq 0$ and $\lambda_{0,0}^{(0)}+\lambda_{0,0}^{(1)}=1$,

$$
(-1)^{r_{1}+r_{2}} \Delta_{1}^{r_{1}} \Delta_{2}^{r_{2}} \lambda_{j, k}^{\left(b_{0}\right)} \geq 0,0 \leq j+r_{1} \leq t_{00}+t_{01}, 0 \leq k+r_{2} \leq t_{11}+t_{10} .
$$


An infinite bivariate sequence $\{\bar{\lambda}(i, j): i, j=0,1, \ldots\}$ with $\bar{\lambda}(0,0)=1$ is called infinite rectangular completely monotone (IRCM) if $\bar{\lambda}(i, j)$ satisfies (5) for all nonnegative integers $i, j$ and $r_{1}, r_{2}$. IRCM characterizes partial exchangeability as stated in the following theorem. The simple proof is based on the well-known result in the two-dimensional Hausdorff momentum problem by Haviland (1935) and is relegated in the Appendix. This characterization is useful in obtaining parsimonious mixtures of Markov chains, see Peng, Dang and Garner (2009).

Theorem 2 Two infinite sequences $\left\{\bar{\lambda}^{\left(b_{0}\right)}(i, j): i, j=0,1, \ldots\right\}$ for $b_{0}=0,1$ satisfying $\bar{\lambda}^{(0)}(0,0)+\bar{\lambda}^{(1)}(0,0)=1$ induce a probability distribution (2) if and only if they are IRCM.

Clearly the matrix $\mathbf{t}=\left\{t_{i j}: i, j=0,1\right\}$ of transition counts and the initial state $b_{0}$ are sufficient statistics of the PE distribution. The number of the strings $B_{0}, B_{1}, \ldots, B_{n}$ that have a common transition matrix $\mathbf{t}$ is $\left(\begin{array}{c}n_{0}-1 \\ t_{00}\end{array}\right)\left(\begin{array}{c}n_{1}-1 \\ t_{11}\end{array}\right)$, where $n_{0}, n_{1}$ are the number of zero's and one's respectively. See Quintana and Newton (1998) for details. Let $p\left(\mathbf{t}, b_{0} ; \boldsymbol{\lambda}\right)$ be the probability that $B_{0}, B_{1}, \ldots, B_{n}$ have a common transition matrix $\mathbf{t}$ with initial state $b_{0}$. Then by (2),

$$
p\left(\mathbf{t}, b_{0} ; \boldsymbol{\lambda}\right)=\left(\begin{array}{c}
n_{0}-1 \\
t_{00}
\end{array}\right)\left(\begin{array}{c}
n_{1}-1 \\
t_{11}
\end{array}\right) \sum_{i=0}^{t_{01}} \sum_{j=0}^{t_{10}}(-1)^{i+j}\left(\begin{array}{c}
t_{01} \\
i
\end{array}\right)\left(\begin{array}{c}
t_{10} \\
j
\end{array}\right) \lambda_{t_{00}+i, t_{11}+j}^{\left(b_{0}\right)} .
$$

This formula seems to suggest that the possible values of $t_{00}$ and $t_{11}$ with which $\left\{p\left(\mathbf{t}, b_{0} ; \boldsymbol{\lambda}\right): b_{0}=0,1, \forall \mathbf{t}\right\}$ constitutes a probability distribution are $\left\{0, \ldots, n_{0}-1\right\}$ and $\left\{0, \ldots, n_{1}-1\right\}$ respectively. An further glance reveals that the constraints on $t_{01}, t_{10}$ are not taken into account, that is, $t_{01}, t_{10}$ can take at most half the value of the length $n+1$ of the string because the number of transitions from zero to one or from one to zero is determined by $n_{0} \wedge n_{1}=\min \left(n_{0}, n_{1}\right)$. Indeed, the values of $t_{01}$ and $t_{10}$ are equal if the initial state $b_{0}$ and terminal state $b_{n}$ are identical $b_{0}=b_{n}$, otherwise they differ by one, i.e., $t_{01}-1=t_{10}$ if $b_{0}=0, b_{n}=1$ and $t_{10}-1=t_{01}$ 
if $b_{0}=1$ and $b_{n}=0$. Thus given the initial and terminal states $b_{0}, b_{n}$, either of $t_{01}$ and $t_{10}$ determines the other. Note that the length of the string $B_{0}, \ldots, B_{n}$ can be calculated by $n+1=t_{00}+t_{01}+t_{10}+t_{11}+1$. Also $n_{0}-\mathbf{1}\left[b_{0}=0\right]=t_{00}+t_{10}$, $n_{1}-\mathbf{1}\left[b_{0}=1\right]=t_{11}+t_{01}$, and $n_{0}+n_{1}=n+1$. Thus the pentuple $\left(\mathbf{t}, b_{0}\right)$ can be equivalently expressed as the pentuple $\left(s, n_{0}, b_{0}, b_{n}, n+1\right)$ where $s$ is either $t_{01}$ or $t_{10}$. In fact, the latter is more convenient in describing the distribution. For this reason, we shall write $p\left(\mathbf{t}, b_{0} ; \boldsymbol{\lambda}\right)$ as $p\left(s, n_{0}, b_{0}, b_{n}, n+1 ; \boldsymbol{\lambda}\right)$ or as usual omit $n+1$ and write as $p\left(s, n_{0}, b_{0}, b_{n} ; \boldsymbol{\lambda}\right)$. We now derive the formula for $p\left(s, n_{0}, b_{0}, b_{n} ; \boldsymbol{\lambda}\right)$. To simplify our description, let us consider the string starting with state $b_{0}=0$ and denote $s=t_{01}$. Then $n_{0}-1=t_{00}+t_{10}, n_{1}=t_{11}+t_{01}$, and $n_{1}=n+1-n_{0}$. There are now two cases. Case 1. The terminal state is 0 . Then $t_{01}=t_{10}=s, t_{00}=n_{0}-1-s, t_{11}=n_{1}-s$. The range of $s$ is the integers in the interval $\left[0,\left(n_{0}-1\right) \wedge n_{1}\right]$.

Case 2. The terminal state is 1 . Then $t_{01}=s, t_{10}=s-1, t_{00}=n_{0}-s, t_{11}=n_{1}-s$. The range of $s$ is the integers in the interval $\left[1, n_{0} \wedge n_{1}\right]$.

Accordingly, we have

$$
\begin{gathered}
p\left(s, n_{0}, 0, b_{n} ; \boldsymbol{\lambda}\right)=\left(\begin{array}{c}
n_{0}-1 \\
s-\mathbf{1}_{1}
\end{array}\right)\left(\begin{array}{c}
n_{1}-1 \\
s-1
\end{array}\right) \times \\
\sum_{i=0}^{s} \sum_{j=0}^{s-\mathbf{1}_{1}}(-1)^{i+j}\left(\begin{array}{c}
s \\
i
\end{array}\right)\left(\begin{array}{c}
s-\mathbf{1}_{1} \\
j
\end{array}\right) \lambda_{n_{0}-\mathbf{1}_{0}-s+i, n_{1}-s+j}^{(0)}, \\
b_{n}=0,1, n_{0}=1, \ldots, n+1, s=\mathbf{1}_{1}, \ldots,\left(n_{0}-\mathbf{1}_{0}\right) \wedge n_{1} .
\end{gathered}
$$

where $\mathbf{1}_{1}=\mathbf{1}\left[b_{n}=1\right]$ and $\mathbf{1}_{0}=1-\mathbf{1}_{1}$. Here we set $\left(\begin{array}{c}m \\ i\end{array}\right)=0$ if either $i>m$, or $m<0$ or $i<0$ except $\left(\begin{array}{c}m \\ i\end{array}\right)=1$ when $m=i \in\{0,-1\}$. Analogously one has the formula for the string starting with 1 . The two formulas can be combined in one as follows:

$$
\begin{gathered}
p\left(s, n_{0}, b_{0}, b_{n} ; \boldsymbol{\lambda}\right)=\left(\begin{array}{c}
n_{0}-1 \\
s-1+\mathbf{1}_{00}
\end{array}\right)\left(\begin{array}{c}
n_{1}-1 \\
s-1+\mathbf{1}_{11}
\end{array}\right) \times \\
\sum_{i=0}^{s-\mathbf{1}_{10}} \sum_{j=0}^{s-\mathbf{1}_{01}}(-1)^{i+j}\left(\begin{array}{c}
s-\mathbf{1}_{10} \\
i
\end{array}\right)\left(\begin{array}{c}
s-\mathbf{1}_{01} \\
j
\end{array}\right) \lambda_{n_{00}-s+i, n_{11}-s+j}^{\left(b_{0}\right)} \\
b_{0}, b_{n}=0,1, n_{0}=\mathbf{i}_{0}, \ldots, n+1-\mathbf{i}_{1}, s=\mathbf{1}_{01}+\mathbf{1}_{10}, \ldots, n_{00} \wedge n_{11},
\end{gathered}
$$


where $\mathbf{1}_{i j}=\mathbf{1}\left[b_{0}=i, b_{n}=j\right], i, j=0,1, n_{00}=n_{0}-\mathbf{1}_{00}, n_{11}=n_{1}-\mathbf{1}_{11}, \mathbf{i}_{0}=\mathbf{1}\left[b_{0}=0\right]$ and $\mathbf{i}_{1}=1-\mathbf{i}_{0}$. Since the sum of all the probabilities $P\left(B_{0}=b_{0}, B_{1}=b_{1}, \ldots, B_{n}=b_{n}\right)$ for $b_{0}, \ldots, b_{n}=0,1$ is one, it follows

$$
\sum_{b_{0}=0}^{1} \sum_{b_{n}=0}^{1} \sum_{n_{0}=\mathbf{i}_{0}}^{n+1-\mathbf{i}_{1}} \sum_{s=\mathbf{1}_{01}+\mathbf{1}_{10}}^{n_{00} \wedge n_{11}} p\left(s, n_{0}, b_{0}, b_{n} ; \boldsymbol{\lambda}\right)=1 .
$$

This shows the following theorem.

Theorem $3\left\{p\left(s, n_{0}, b_{0}, b_{n} ; \boldsymbol{\lambda}\right): b_{0}, b_{n}=0,1, n_{0}=\mathbf{i}_{0}, \ldots, n+1-\mathbf{i}_{1}, s=\mathbf{1}_{01}+\right.$ $\left.\mathbf{1}_{10}, \ldots, n_{00} \wedge n_{11}\right\}$ is a probability distribution.

The justification that $p\left(s, n_{0}, b_{0}, b_{n} ; \boldsymbol{\lambda}\right)$ constitutes a probability distribution provides the theoretical basis for the statistical inference with it, such as the use of the log likelihood as a criterion of model selection, see the application of the distribution in real data by Peng, et al. (2009).

From the above discussion, it is easily checked these relations:

$$
s=t_{01} \mathbf{i}_{0}+t_{10} \mathbf{i}_{1}, \quad n_{0}=t_{00}+t_{10}-\mathbf{i}_{0}, \quad b_{n}=\mathbf{1}\left[t_{01}=t_{10}\right] b_{0}+\mathbf{1}\left[t_{01}=t_{10}+1\right] .
$$

Let $S=B_{0}+\ldots+B_{n}$. Then an immediate consequence of the above theorem is

$$
\mathbb{P}\left(S=n+1-n_{0}\right)=\sum_{b_{0}=0}^{1} \sum_{b_{n}=0}^{1} \sum_{s=\mathbf{1}_{01}+\mathbf{1}_{10}}^{n_{00} \wedge n_{11}} p\left(s, n_{0}, b_{0}, b_{n} ; \boldsymbol{\lambda}\right), \quad n_{0}=0,1, \ldots, n+1 .
$$

This generalizes the binomial distribution under exchangeability and hence under independence (binomial). The proof of (11) is a probabilistic one. An algebraic proof is interesting, gives additional insight and can be found in the Appendix.

\section{Appendix: Technical Details}

Proof of Theorem 2. We only have to show the sufficiency. It follows from the case 6 (page 168) of the theorem in Haviland (1935) that an infinite sequence $\{\bar{\lambda}(i, j)\}$ is 
IRCM if and only if it can be represented as

$$
\bar{\lambda}(i, j)=\int_{0}^{\infty} \int_{0}^{\infty} e^{-i x-j y} d \bar{P}(x, y), \quad i, j=0,1, \ldots
$$

for a probability $\bar{P}$ on $[0, \infty)^{2}$. An application of the result to $\left\{\bar{\lambda}\left(b_{0}, i, j\right): i, j=\right.$ $0,1, \ldots\}$ for $b_{0}=0,1$ and substitution of the representations in (4) yields the desired result.

Proof of Theorem 3. Using the integral representation (1), we have surprisingly found that we can prove the following stronger result:

$$
q_{n+1}\left(u, v \mid b_{0}\right) \equiv 1, \quad b_{0}=0,1,0 \leq u, v \leq 1
$$

where $q_{n+1}\left(u, v \mid b_{0}\right)$ equals

$$
\sum_{b_{n}=0}^{1} \sum_{n_{0}=\mathbf{i}_{0}}^{n+1} \sum_{s=\mathbf{1}_{01}+\mathbf{1}_{10}}^{n_{00} \wedge n_{11}}\left(\begin{array}{c}
n_{0}-1 \\
s-1+\mathbf{1}_{00}
\end{array}\right)\left(\begin{array}{c}
n_{1}-1 \\
s-1+\mathbf{1}_{11}
\end{array}\right) u^{n_{00}-s}(1-u)^{s-\mathbf{1}_{10}} v^{n_{11}-s}(1-v)^{s-\mathbf{1}_{01}}
$$

The identity (13) certainly implies (11). In addition, it is noteworthy to mention that (13) is an identity which generalizes the binomial identity, i.e., when $u+v=1$, it simplifies to the binomial identity

$$
\sum_{n_{0}=0}^{n+1}\left(\begin{array}{c}
n+1 \\
n_{0}
\end{array}\right) u^{n_{0}} v^{n+1-n_{0}}=(u+v)^{n+1}=1 .
$$

We shall prove (13) for $b_{0}=0$ and the case for $b_{0}=1$ holds by symmetry. Let $F_{n}(u, v)=q_{n+1}\left(u, v \mid b_{0}=0\right)$. Then (13) immediately follows from the iterative formula:

$$
F_{n}(u, v)=u F_{n-1}(u, v)+(1-u) F_{n-1}(v, u), \quad 0 \leq u, v \leq 1, n=1,2, \ldots
$$


We shall prove this by mathematical induction. For $n=2 k$, we have

$$
\begin{aligned}
F_{2 k}(u, v) & =\sum_{m=1}^{k} \sum_{s=0}^{m-1}\left(\begin{array}{c}
m-1 \\
s
\end{array}\right)\left(\begin{array}{c}
2 k-m \\
s-1
\end{array}\right) u^{m-1-s}(1-u)^{s} v^{2 k+1-m-s}(1-v)^{s} \\
& +\sum_{m=k+1}^{2 k+1} \sum_{s=0}^{2 k+1-m}\left(\begin{array}{c}
m-1 \\
s
\end{array}\right)\left(\begin{array}{c}
2 k-m \\
s-1
\end{array}\right) u^{m-1-s}(1-u)^{s} v^{2 k+1-m-s}(1-v)^{s} \\
& +\sum_{m=1}^{k} \sum_{s=0}^{m}\left(\begin{array}{c}
m-1 \\
s-1
\end{array}\right)\left(\begin{array}{c}
2 k-m \\
s-1
\end{array}\right) u^{m-s}(1-u)^{s} v^{2 k+1-m-s}(1-v)^{s-1} \\
& +\sum_{m=k+1}^{2 k} \sum_{s=0}^{2 k+1-m}\left(\begin{array}{c}
m-1 \\
s-1
\end{array}\right)\left(\begin{array}{c}
2 k-m \\
s-1
\end{array}\right) u^{m-s}(1-u)^{s} v^{2 k+1-m-s}(1-v)^{s-1}
\end{aligned}
$$

Keep in mind that we make use of the convention $\left(\begin{array}{c}m \\ i\end{array}\right)=0$ if either $i>m$, or $m<0$ or $i<0$ except $\left(\begin{array}{c}m \\ i\end{array}\right)=1$ when $m=i \in\{0,-1\}$. For $n=2 k+1$, we have

$$
\begin{aligned}
F_{2 k+1}(u, v) & =\sum_{m=1}^{k+1} \sum_{s=0}^{m-1}\left(\begin{array}{c}
m-1 \\
s
\end{array}\right)\left(\begin{array}{c}
2 k+1-m \\
s-1
\end{array}\right) u^{m-1-s}(1-u)^{s} v^{2 k+2-m-s}(1-v)^{s} \\
& +\sum_{m=k+2}^{2 k+2} \sum_{s=0}^{2 k+2-m}\left(\begin{array}{c}
m-1 \\
s
\end{array}\right)\left(\begin{array}{c}
2 k+1-m \\
s-1
\end{array}\right) u^{m-1-s}(1-u)^{s} v^{2 k+2-m-s}(1-v)^{s} \\
& +\sum_{m=1}^{k+1} \sum_{s=0}^{m}\left(\begin{array}{c}
m-1 \\
s-1
\end{array}\right)\left(\begin{array}{c}
2 k+1-m \\
s-1
\end{array}\right) u^{m-s}(1-u)^{s} v^{2 k+2-m-s}(1-v)^{s-1} \\
& +\sum_{m=k+2}^{2 k+1} \sum_{s=0}^{2 k+2-m}\left(\begin{array}{c}
m-1 \\
s-1
\end{array}\right)\left(\begin{array}{c}
2 k+1-m \\
s-1
\end{array}\right) u^{m-s}(1-u)^{s} v^{2 k+2-m-s}(1-v)^{s-1} .
\end{aligned}
$$

By the identity $\left(\begin{array}{l}n \\ s\end{array}\right)=\left(\begin{array}{c}n-1 \\ s\end{array}\right)+\left(\begin{array}{c}n-1 \\ s-1\end{array}\right)$, we have $F_{2 k+1}(u, v)=D_{1}+D_{2}$, where

$$
\begin{aligned}
D_{1} & =\sum_{m=1}^{k+1} \sum_{s=0}^{m-1}\left(\begin{array}{c}
m-2 \\
s
\end{array}\right)\left(\begin{array}{c}
2 k+1-m \\
s-1
\end{array}\right) u^{m-1-s}(1-u)^{s} v^{2 k+2-m-s}(1-v)^{s} \\
& +\sum_{m=k+2}^{2 k+2} \sum_{s=0}^{2 k+2-m}\left(\begin{array}{c}
m-2 \\
s
\end{array}\right)\left(\begin{array}{c}
2 k+1-m \\
s-1
\end{array}\right) u^{m-1-s}(1-u)^{s} v^{2 k+2-m-s}(1-v)^{s} \\
& +\sum_{m=1}^{k+1} \sum_{s=0}^{m}\left(\begin{array}{c}
m-2 \\
s-1
\end{array}\right)\left(\begin{array}{c}
2 k+1-m \\
s-1
\end{array}\right) u^{m-s}(1-u)^{s} v^{2 k+2-m-s}(1-v)^{s-1} \\
& +\sum_{m=k+2}^{2 k+1} \sum_{s=0}^{2 k+2-m}\left(\begin{array}{c}
m-2 \\
s-1
\end{array}\right)\left(\begin{array}{c}
2 k+1-m \\
s-1
\end{array}\right) u^{m-s}(1-u)^{s} v^{2 k+2-m-s}(1-v)^{s-1}
\end{aligned}
$$




$$
\begin{aligned}
D_{2} & =\sum_{m=1}^{k+1} \sum_{s=0}^{m-1}\left(\begin{array}{c}
m-2 \\
s-1
\end{array}\right)\left(\begin{array}{c}
2 k+1-m \\
s-1
\end{array}\right) u^{m-1-s}(1-u)^{s} v^{2 k+2-m-s}(1-v)^{s} \\
& +\sum_{m=k+2}^{2 k+1} \sum_{s=0}^{2 k+2-m}\left(\begin{array}{c}
m-2 \\
s-1
\end{array}\right)\left(\begin{array}{c}
2 k+1-m \\
s-1
\end{array}\right) u^{m-1-s}(1-u)^{s} v^{2 k+2-m-s}(1-v)^{s} \\
& +\sum_{m=1}^{k+1} \sum_{s=0}^{m}\left(\begin{array}{c}
m-2 \\
s-2
\end{array}\right)\left(\begin{array}{c}
2 k+1-m \\
s-1
\end{array}\right) u^{m-s}(1-u)^{s} v^{2 k+2-m-s}(1-v)^{s-1} \\
& +\sum_{m=k+2}^{2 k+1} \sum_{s=0}^{2 k+2-m}\left(\begin{array}{c}
m-2 \\
s-2
\end{array}\right)\left(\begin{array}{c}
2 k+1-m \\
s-1
\end{array}\right) u^{m-s}(1-u)^{s} v^{2 k+2-m-s}(1-v)^{s-1}
\end{aligned}
$$

By the change $m-1 \rightarrow m$ of the variable, we have

$$
\begin{aligned}
D_{1}= & \sum_{m=1}^{k} \sum_{s=0}^{m}\left(\begin{array}{c}
m-1 \\
s
\end{array}\right)\left(\begin{array}{c}
2 k-m \\
s-1
\end{array}\right) u^{m-s}(1-u)^{s} v^{2 k+1-m-s}(1-v)^{s} \\
+ & \sum_{m=k+1}^{2 k+1} \sum_{s=0}^{2 k+1-m}\left(\begin{array}{c}
m-1 \\
s
\end{array}\right)\left(\begin{array}{c}
2 k-m \\
s-1
\end{array}\right) u^{m-s}(1-u)^{s} v^{2 k+1-m-s}(1-v)^{s} \\
+ & \sum_{m=1}^{k} \sum_{s=0}^{m+1}\left(\begin{array}{c}
m-1 \\
s-1
\end{array}\right)\left(\begin{array}{c}
2 k-m \\
s-1
\end{array}\right) u^{m+1-s}(1-u)^{s} v^{2 k+1-m-s}(1-v)^{s-1} \\
+ & \sum_{m=k+1}^{2 k} \sum_{s=0}^{2 k+1-m}\left(\begin{array}{c}
m-1 \\
s-1
\end{array}\right)\left(\begin{array}{c}
2 k-m \\
s-1
\end{array}\right) u^{m+1-s}(1-u)^{s} v^{2 k+1-m-s}(1-v)^{s-1}=u F_{2 k}(u, v) \\
& D_{2}=\sum_{m=0}^{k} \sum_{s=0}^{m}\left(\begin{array}{c}
m-1 \\
s-1
\end{array}\right)\left(\begin{array}{c}
2 k-m \\
s-1
\end{array}\right) u^{m-s}(1-u)^{s} v^{2 k+1-m-s}(1-v)^{s} \\
& +\sum_{m=k+1}^{2 k} \sum_{s=0}^{2 k-m+1}\left(\begin{array}{c}
m-1 \\
s-1
\end{array}\right)\left(\begin{array}{c}
2 k-m \\
s-1
\end{array}\right) u^{m-s}(1-u)^{s} v^{2 k+1-m-s}(1-v)^{s} \\
& +\sum_{m=1}^{k} \sum_{s=0}^{m}\left(\begin{array}{c}
m-1 \\
s-1
\end{array}\right)\left(\begin{array}{c}
2 k-m \\
s
\end{array}\right) u^{m-s}(1-u)^{s+1} v^{2 k-m-s}(1-v)^{s} \\
& +\sum_{m=k+1}^{2 k} \sum_{s=0}^{2 k-m}\left(\begin{array}{c}
m-1 \\
s-1
\end{array}\right)\left(\begin{array}{c}
2 k-m \\
s
\end{array}\right) u^{m-s}(1-u)^{s+1} v^{2 k-m-s}(1-v)^{s}
\end{aligned}
$$


Again by the change $l=2 k+1-m \rightarrow m$ of the variable, we have

$$
\begin{aligned}
D_{2} & =\sum_{l=k+1}^{2 k+1} \sum_{s=0}^{2 k+1-l}\left(\begin{array}{c}
2 k-l \\
s-1
\end{array}\right)\left(\begin{array}{l}
l-1 \\
s-1
\end{array}\right) v^{l-s}(1-v)^{s} u^{2 k+1-l-s}(1-u)^{s} \\
& +\sum_{l=1}^{k} \sum_{s=0}^{l}\left(\begin{array}{c}
2 k-l \\
s-1
\end{array}\right)\left(\begin{array}{c}
l-1 \\
s-1
\end{array}\right) v^{l-s}(1-v)^{s} u^{2 k+1-l-s}(1-u)^{s} \\
& +\sum_{l=k+1}^{2 k+1} \sum_{s=0}^{2 k+1-l}\left(\begin{array}{c}
2 k-l \\
s-1
\end{array}\right)\left(\begin{array}{c}
l-1 \\
s
\end{array}\right) v^{l-1-s}(1-v)^{s} u^{2 k+1-l-s}(1-u)^{s+1} \\
& +\sum_{l=1}^{k} \sum_{s=0}^{l-1}\left(\begin{array}{c}
2 k-l \\
s-1
\end{array}\right)\left(\begin{array}{c}
l-1 \\
s
\end{array}\right) v^{l-1-s}(1-v)^{s} u^{2 k+1-l-s}(1-u)^{s+1}=(1-u) F_{2 k}(v, u) .
\end{aligned}
$$

Thus the iterative formula (14) holds for odd $n$. Analogously, it holds for even $n$.

\section{References}

[1] Chow, Y. S. and Teicher, H. (1997). Probability Theory: Independence, Interchangeability, Martingales, 3rd ed., Springer.

[2] Dang, X., Keeton, S. L. and Peng, H. (2009). "A Unified Approach for Analyzing Exchangeable Binary Data with Applications to Clinical and Developmental Toxicity Studies.", Statist. Med., Accepted in December, 2008, Available at URL: http://www.math.iupui.edu/ hpeng.

[3] Diaconis, P. (1988). "Recent Progress on de Finetti's Notion of Exchangeability" in Bayesian Statistics 3,111-125, eds. J. M. Bernardo, M. H. De Groot, D. V. Lindley and A. F. M. Smith, Oxford University Press, Oxford, UK.

[4] Diaconis, P. and Freedman, D. (1980a). "de Finetti's Theorem for Markov Chains." Ann. Probabil. 8, 115-130.

[5] Diaconis, P. and Freedman, D. (1980b). "de Finetti's Generalizations of Exchangeability." in Studies in Inductive Logic ond Probability 11, ed. R. C. Jeffrey, Berkeley, University of California Press. 
[6] Diaconis, P. and Freedman, D. (1980c). "Finite Exchangeable Sequences". Ann. Probabil. 8, 745-764.

[7] Freedman, D. (1962a). "Mixtures of Markov Processes." Ann. Math. Statist. 33, 114-118.

[8] Freedman, D. (1962b). "Invariants Under Mixing Which Generalize de Finetti's Theorem." Ann. Math. Statist. 33, 916-923.

[9] George, E. O. and Bowman, D. (1995). "A Full Likelihood Procedure for Analysing Exchangeable Binary Data." Biometrics 51, 512-523.

[10] Gupta, J. C. (1999). "The Moment Problem for the Standard k-Dimensional Simplex." Sankhya, Ser. A, 61, 286-291.

[11] Haviland, E. K. (1935). "On the Momentum Problem for Distribution Functions in More than One Dimension." Amer. J. Math. 57, 562-568.

[12] Kendall, D. G. (1967). "On Finite and Infinite Sequences of Exchangeable Events." Studia Scientiarum Mathematicarum Hungarica 2, 319-327.

[13] Peng, H., Dang, X. and Garner, L. (2009). "Analyzing Partially Exchangeable Data", Manuscript, Available at URL: http://www.math.iupui.edu/ hpeng.

[14] Quintana, F. A. and Newton, M. A. (1998). "Assessing the Order of Dependence for Partially Exchangeable Binary Data." J. Amer. Statist. Asso. 93, 194-202.

[15] Zaman, A. (1984). "Urn Models for Markov Exchangeability." Ann. Probabil. 11, 223-229. 